\title{
Co-Design of Event Generator and Dynamic Output Feedback Controller for LTI Systems
}

\author{
Dan Ma, ${ }^{1}$ Junxian Han, ${ }^{1}$ Dali Zhang, ${ }^{2}$ and Yanjun Liu ${ }^{3}$ \\ ${ }^{1}$ State Key Laboratory of Synthetical Automation for Process Industries, College of Information Science and Engineering, \\ Northeastern University, Shenyang 110819, China \\ ${ }^{2}$ School of Aeronautics and Astronautics, Dalian University of Technology, Dalian 116024, China \\ ${ }^{3}$ College of Science, Liaoning University of Technology, Jinzhou, Liaoning 121001, China
}

Correspondence should be addressed to Dan Ma; madan@mail.neu.edu.cn

Received 8 July 2014; Revised 15 August 2014; Accepted 18 August 2014

Academic Editor: Xi-Ming Sun

Copyright (C) 2015 Dan Ma et al. This is an open access article distributed under the Creative Commons Attribution License, which permits unrestricted use, distribution, and reproduction in any medium, provided the original work is properly cited.

This paper presents a co-design method of the event generator and the dynamic output feedback controller for a linear timeinvariant (LIT) system. The event-triggered condition on the sensor-to-controller and the controller-to-actuator depends on the plant output and the controller output, respectively. A sufficient condition on the existence of the event generator and the dynamic output feedback controller is proposed and the co-design problem can be converted into the feasibility of linear matrix inequalities (LMIs). The LTI system is asymptotically stable under the proposed event-triggered controller and also reduces the computing resources with respect to the time-triggered one. In the end, a numerical example is given to illustrate the effectiveness of the proposed approach.

\section{Introduction}

With plenty of control applications on the digital platforms, event-triggered control becomes more and more popular due to its advantages on control systems with limited resources. For networked control systems, most literature focuses on the performance analysis of the system under the network bandwidth limitation; see [1-4]. However, less traffic requirement, better resource utilization, and better steady-state performance are all the significant factors. Compared with timetriggered control, event-triggered control has its advantages. In the traditional digital control techniques, the controller updates periodically. Analysis and synthesis of the system by using the periodic sampling is much easier than using the aperiodic sampling. However, the periodic sampling leads to a waste of computation and communication resources sometimes. From the resource allocation point of view, the event-triggered control was proposed; see the literature [57] and references therein. Event-triggered control is a control scheme in which the controller updates as long as the system state (or output) satisfies a well-designed condition.
The condition is called event generator, which can maintain the necessary properties of the system, such as convergence and stability. By using event-triggered control, computation and communication resources will be utilized only when necessary. Therefore, the event-triggered mechanism is a kind of "on-demand" executive strategy and can guarantee the performance of the system as well.

Due to the advantage of reducing computation and communication resources, event-triggered control has been taken more and more into consideration. It is first presented by Dorf in [5]; several different event-triggered schemes are investigated for a variety of systems with control performances. In $[6,7]$, the authors experimentally demonstrate the saving resources of event-triggered control while simultaneously preserving the performance of the system. Event-triggered control for the first-order linear stochastic system forces the output variance to be considerably smaller with respect to periodic control [6]. A simple event-based PID controller is studied in [7], which contains a time-triggered event detector and an event-triggered PID controller. The PID controller cannot calculate the control signal unless the variable from 
the event detector has enough changes. An asynchronous emulation-based event-triggered feedback approach is taken into account in [8], where a directly digital design strategy is included. In [9], Tabuada proposes an event-triggered mechanism for nonlinear system to guarantee the asymptotical stability of the system and relax the traditional periodic execution requirements. The method proposed in [9] is extended to the exponential input-to-state stability (ISS) in [10]. In [11], Lunze and Lehmann propose a method on eventbased state-feedback control for linear systems, with bounded disturbances, in which the sensitivity bound of the event generator can be chosen such that the event-triggered control system approximates the continuous state-feedback control loop.

However, not all of the system states can be directly measured in applications; the event-triggered control based on the system output (a part of the system state information) is much more practical; see, for example, [12-16]. Decentralized event-triggered mechanism is applied to the dynamic output feedback control system in [12]. For guaranteeing the $L_{\infty}$ performance, the event generator is designed and the bound of interevent time is also provided. The optimal control problem under the event-triggered output feedback control is studied for discrete-time systems in [13] and an upper bound on the optimal cost is presented. The inputto-state stability under the self-triggered dynamic output feedback control is studied in [14], where a discrete-time observer is in cascade with a full state-feedback self-triggered controller. The designed event generator renders the ISS of the system with respect to exogenous disturbances. The result is extended to the decentralized event-triggered system in [15]. In [16], the event-triggered conditions are proposed for three kinds of event-triggered dynamic output feedback control architectures, in which a global lower bound on the intersample times is also provided to guarantee the asymptotic stability of the closed-loop system. However, most work in the literature focuses on designing the event generator, does not mention the continuous control algorithm. Most of them assume that the controller has been designed previously. But in fact, the event-triggered controller includes both the event generator and the controller. How to co-design the event generator and the controller simultaneously is much more challengeable. $\mathrm{Li}$ and $\mathrm{Xu}[17,18]$ give us a co-design method for both discrete-time linear systems and LPV systems under the state feedback controller. While co-designing the event generator and the controller based on the system output is quite significant from the practical application point of view. To the best of the authors' knowledge, co-designing of event generator and the dynamic output feedback controller has not been investigated. Comparing to the static state feedback controller, the dynamic output feedback controller can often provide more adjustable parameters. It is a challenge to codesign the event generator and the dynamic output feedback controller as well.

The remainder of the paper is organized as follows. In Section 2, the necessary notations and preliminaries are provided. The problem statement of event-triggered dynamic output feedback control is presented in Section 3. Section 4 presents a sufficient condition on how to co-design the event

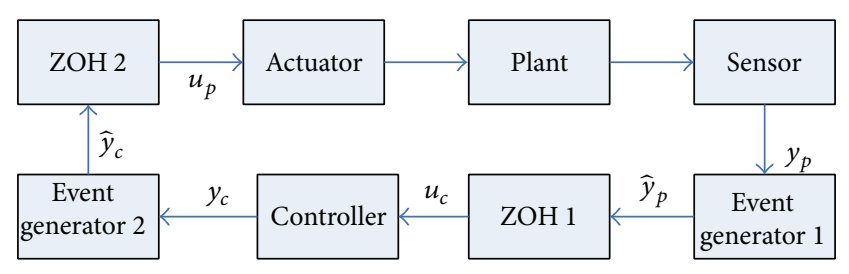

FIGURE 1: Event-triggered dynamic output feedback control loop.

generator and the dynamic output feedback controller, under which the linear system is asymptotically stable. Numerical simulation results are given in Section 5, which verify the effectiveness of the proposed methods. Finally, conclusions are included in Section 6.

\section{Preliminaries}

The notation $\|\cdot\|$ is used to denote the Euclidean norm. $X>$ $0(X<0)$ represents that $X$ is a positive (negative) definite matrix. $X^{T}$ and $X^{-1}$ denote the transpose and the inverse of matrix $X$. I represents the unit matrix with appropriate dimensions; $Z_{0}{ }^{+}$is the set of all nonnegative integers. For simplicity, the form $\left(\begin{array}{ll}A & B \\ * & C\end{array}\right)$ is equivalent to the symmetric matrix of the form $\left(\begin{array}{c}A \\ A^{T} C \\ B^{T}\end{array}\right)$. In what follows, if not explicitly stated, matrices are assumed to have compatible dimensions.

The following lemma will be used in the proof of the main result.

Lemma 1 (see [19]). Consider a symmetric matrix $S=$ $\left[\begin{array}{ll}S_{11} & S_{12} \\ S_{21} & S_{22}\end{array}\right]$, where $S_{11} \in R^{r \times r}, S_{12} \in R^{r \times(n-r)}, S_{21} \in R^{(n-r) \times r}, S_{22} \in$ $R^{(n-r) \times(n-r)}$. Then $S<0$ only if $S_{11}<0$ and $S_{22}-S_{12}^{T} S_{11}^{-1} S_{12}<0$ or equivalently, $S_{22}<0$ and $S_{11}-S_{12} S_{22}^{-1} S_{12}^{T}<0$.

\section{Problem Statement}

A linear plant controlled by an event-triggered dynamic output feedback controller is shown in Figure 1. The event generator is an implementation of the event-triggered condition. We need to design an output-dependent eventtriggered condition which can be used to decide when the measurement and the control law update. If the output signal $y_{p}$ measured from the sensor satisfies the event-triggered condition 1, it will be sent to the controller through the zeroorder-hold 1 ( $\mathrm{ZOH} 1)$; otherwise it will not be sent. Similarly, if the output of the controller $y_{c}$ satisfies the event-triggered condition 2, the output of the controller will be sent to the actuator through the $\mathrm{ZOH} \mathrm{2;}$ otherwise it does not transmit. Here we denote the triggered instant by $t_{i}, i \in Z_{0}^{+}$.

We consider a linear time-invariant plant given by

$$
\begin{gathered}
\dot{x}_{p}(t)=A_{p} x_{p}(t)+B_{p} u_{p}(t), \quad x_{p}(0)=x_{p 0}, \\
y_{p}(t)=C_{p} x_{p}(t),
\end{gathered}
$$

where $x_{p}(t) \in R^{n}$ denotes the plant state; $x_{p}(0)=x_{p 0}$ is the initial state of the plant; $u_{p}(t) \in R^{m}$ is the plant input; 
$y_{p}(t) \in R^{q}$ is the output of the plant. $A_{p}, B_{p}$, and $C_{p}$ are the known constant matrices with appropriate dimensions.

The plant is controlled by a dynamic output feedback controller given by

$$
\begin{gathered}
\dot{x}_{c}(t)=A_{c} x_{c}(t)+B_{c} u_{c}(t), \quad x_{c}(0)=x_{c 0}, \\
y_{c}(t)=C_{c} x_{c}(t),
\end{gathered}
$$

where $x_{c}(t) \in R^{n}$ denotes the controller state; $x_{c}(0)=x_{c 0}$ is the initial state of the controller; $u_{c}(t) \in R^{q}$ is the controller input; $y_{c}(t) \in R^{m}$ is the output of the controller. $A_{c}, B_{c}$, and $C_{c}$ are matrices appropriate dimensions, which will be designed in the following part.

Whether $y_{p}(t)$ and $y_{c}(t)$ are sent or not is up to the event generator conditions. That is to say, the controller cannot calculate the control signal unless $y_{p}$ and $y_{c}$ in the event generator have enough change. Since $t_{i}, i \in Z_{0}^{+}$is the triggered instant, $y_{p}(t)$ and $y_{c}(t)$ are only sent at $t_{i} ; \widehat{y}_{p}(t)$ and $\widehat{y}_{c}(t)$ update at the triggered instants. By using the zero-order holder, $\hat{y}_{p}(t)$ and $\widehat{y}_{c}(t)$ maintain their values until the next triggered instant $t_{i+1}$ arrives. Therefore, $u_{p}(t)=\widehat{y}_{c}(t)$ and $u_{c}(t)=\widehat{y}_{p}(t)$ can be expressed by

$$
\begin{array}{ll}
\widehat{y}_{c}(t)=y_{c}\left(t_{i}\right), & t \in\left[t_{i}, t_{i+1}\right), \\
\widehat{y}_{p}(t)=y_{p}\left(t_{i}\right), \quad t \in\left[t_{i}, t_{i+1}\right) .
\end{array}
$$

Define the errors $e_{p}(t)$ and $e_{c}(t)$ as follows:

$$
\begin{gathered}
e_{p}(t)=\widehat{y}_{p}(t)-y_{p}(t), \\
e_{c}(t)=\widehat{y}_{c}(t)-y_{c}(t) .
\end{gathered}
$$

Combining (1), (2), and (4), we have

$$
\dot{x}=\left(\begin{array}{c}
\dot{x}_{p} \\
\dot{x}_{c}
\end{array}\right)=\left(\begin{array}{cc}
A_{p} & B_{p} C_{c} \\
B_{c} C_{p} & A_{c}
\end{array}\right)\left(\begin{array}{c}
x_{p} \\
x_{c}
\end{array}\right)+\left(\begin{array}{cc}
0 & B_{p} \\
B_{c} & 0
\end{array}\right)\left(\begin{array}{c}
e_{p}(t) \\
e_{c}(t)
\end{array}\right) \text {. }
$$

Thus the closed-loop system can be described by

$$
\dot{x}(t)=\bar{A} x(t)+\bar{B} e(t),
$$

where

$$
\begin{gathered}
\bar{A}=\left(\begin{array}{cc}
A_{p} & B_{p} C_{c} \\
B_{c} C_{p} & A_{c}
\end{array}\right), \quad \bar{B}=\left(\begin{array}{cc}
0 & B_{p} \\
B_{c} & 0
\end{array}\right), \\
e(t)=\left(\begin{array}{c}
e_{p}(t) \\
e_{c}(t)
\end{array}\right), \quad x(t)=\left(\begin{array}{c}
x_{p}(t) \\
x_{c}(t)
\end{array}\right), \\
x_{0}=\left(\begin{array}{l}
x_{p 0} \\
x_{c 0}
\end{array}\right) .
\end{gathered}
$$

We present the following event-triggered condition:

$$
e_{p}^{T} e_{p}+e_{c}^{T} e_{c} \leq \sigma_{1} y_{p}^{T} y_{p}+\sigma_{2} y_{c}^{T} y_{c}, \quad \sigma_{1}>0, \sigma_{2}>0
$$

which means $\widehat{y}_{p}(t)$ is updated once if

$$
\begin{aligned}
& e_{p}^{T}(t) e_{p}(t)+e_{c}^{T}\left(t_{i}\right) e_{c}\left(t_{i}\right) \\
& \quad \leq \sigma_{1} y_{p}^{T}(t) y_{p}(t)+\sigma_{2} y_{c}^{T}\left(t_{i}\right) y_{c}\left(t_{i}\right)
\end{aligned}
$$

is violated and $\widehat{y}_{c}(t)$ is updated once if

$$
\begin{aligned}
& e_{p}^{T}\left(t_{i}\right) e_{p}\left(t_{i}\right)+e_{c}^{T}(t) e_{c}(t) \\
& \quad \leq \sigma_{1} y_{p}^{T}\left(t_{i}\right) y_{p}\left(t_{i}\right)+\sigma_{2} y_{c}^{T}(t) y_{c}(t)
\end{aligned}
$$

is violated for $t \in\left[t_{i}, t_{i+1}\right)$.

The objective of this paper is to co-design the dynamic output feedback controller (2) and the event generator satisfying condition (8) under which the closed-loop system (6) is asymptotically stable.

\section{Main Results}

In this section, we will give a sufficient condition on the existence of the event generator and the dynamic output feedback controller by using LIMs.

Theorem 2. If there exist symmetric positive definite matrices $X \in R^{n \times n}$ and $Y \in R^{n \times n}$, matrices $V \in R^{r \times n}$ and $W \in R^{n \times m}$, and scalars $\delta_{1}>0, \delta_{2}>0$ satisfying the following LMIs

$$
\begin{aligned}
& {\left[\begin{array}{cccc}
Y A_{p}^{T}+A_{p} Y+V^{T} B_{p}^{T}+B_{p} V & Y C_{p}^{T} & V^{T} & B_{p} \\
* & -\delta_{1} I & 0 & 0 \\
* & * & -\delta_{2} I & 0 \\
* & * & * & -I
\end{array}\right]<0,} \\
& {\left[\begin{array}{cccc}
X A_{p}+A_{p}^{T} X+W C_{p}+C_{p}^{T} W^{T} & W & X B_{p} & C_{p}^{T} \\
* & -I & 0 & 0 \\
* & * & -I & 0 \\
* & * & * & -\delta_{1} I
\end{array}\right]<0,}
\end{aligned}
$$

then system (6) under the dynamic output feedback controller (2) and the event-triggered condition (8) is asymptotically stable. The parameters of the controller are as follows:

$$
\begin{gathered}
A_{c}=-\left(Y-X^{-1}\right)\left(A_{P}+Y A_{p}^{T} X+V^{T} B_{p}^{T} X+Y C_{P}^{T} W^{T}\right. \\
\left.+B_{p} B_{p}^{T} X+\delta_{1}^{-1} Y C_{p}^{T} C_{p}\right)^{T} Y^{-1} \\
B_{c}=\left(Y-X^{-1}\right) W \\
C_{c}=V Y^{-1},
\end{gathered}
$$

and the parameters of the event-triggered condition (8) are $\sigma_{1}=$ $\delta_{1}^{-1}, \sigma_{2}=\delta_{2}^{-1}$.

Proof. Choosing a candidate Lyapunov function

$$
V_{x}=x^{T} P x,
$$

we have the following time derivative of $V_{x}$ along with system (6):

$$
\begin{aligned}
\dot{V}_{x} & =2 x^{T} P(\bar{A} x+\bar{B} e) \\
& =x^{T}\left(P \bar{A}+\bar{A}^{T} P\right) x+2 x^{T} P \bar{B} e \\
& \leq x^{T}\left(P \bar{A}+\bar{A}^{T} P+P \bar{B} B^{T} P\right) x+e^{T} e .
\end{aligned}
$$


Letting $V=C_{c} Y$, and from (11), we use Lemma 1 three times and obtain

$$
\begin{aligned}
Y A_{p}^{T} & +A_{p} Y+Y^{T} C_{c}^{T} B_{p}^{T}+\delta_{2}^{-1} Y^{T} C_{c}^{T} C_{c} Y \\
& +\delta_{1}^{-1} Y C_{p}^{T} C_{p} Y+B_{p} C_{c} Y+B_{p} B_{p}^{T}<0 .
\end{aligned}
$$

Similarly, letting $W=\left(Y^{-1}-X\right) B_{c}$ and applying the Schur lemma to LMI (12) three times, we get

$$
\begin{aligned}
X A_{p} & +A_{p}^{T} X+\left(Y^{-1}-X\right) B_{c} C_{p}+C_{p}^{T} B_{c}^{T}\left(Y^{-1}-X\right)^{T} \\
& +X B_{p} B_{p}^{T} X+\delta_{1}^{-1} C_{p}^{T} C_{p} \\
& +\left(Y^{-1}-X\right) B_{c} B_{c}^{T}\left(Y^{-1}-X\right)^{T}<0 .
\end{aligned}
$$

Considering

$$
\begin{aligned}
A_{c}=-\left(Y-X^{-1}\right) & \left(A_{P}+Y A_{p}^{T} X+V^{T} B_{p}^{T} X+Y C_{P}^{T} W^{T}\right. \\
& \left.+B_{p} B_{p}^{T} X+\delta_{1}^{-1} Y C_{p}^{T} C_{p}\right)^{T} Y^{-1}
\end{aligned}
$$

we know that

$$
\begin{aligned}
& Y A_{c}^{T}\left(Y^{-1}-X\right)^{T}+A_{P}+Y A_{p}^{T} X+Y C_{c}^{T} B_{p}^{T} X \\
& \quad+Y C_{P}^{T} B_{c}^{T}\left(Y^{-1}-X\right)^{T}+B_{p} B_{p}^{T} X+\delta_{1}^{-1} Y C_{P}^{T} C_{P}=0 .
\end{aligned}
$$

Combining (16), (17), and (19), we have

$$
\left[\begin{array}{cc}
Q_{1} & Q_{2} \\
* & Q_{3}
\end{array}\right]<0
$$

where

$$
\begin{aligned}
Q_{1}= & Y A_{p}^{T}+A_{p} Y+Y^{T} C_{c}^{T} B_{p}^{T}+B_{p} C_{c} Y+B_{p} B_{p}^{T} \\
& +\delta_{1}^{-1} Y C_{p}^{T} C_{p} Y+\delta_{2}^{-1} Y^{T} C_{c}^{T} C_{c} Y \\
Q_{2}= & Y A_{c}^{T}\left(Y^{-1}-X\right)^{T}+A_{P}+Y A_{p}^{T} X+Y C_{c}^{T} B_{p}^{T} X \\
& +Y C_{P}^{T} B_{c}^{T}\left(Y^{-1}-X\right)^{T}+B_{p} B_{p}^{T} X+\delta_{1}^{-1} Y C_{P}^{T} C_{P}, \\
Q_{3}= & X A_{p}+A_{p}^{T} X+\left(Y^{-1}-X\right) B_{c} C_{p}+C_{p}^{T} B_{c}^{T}\left(Y^{-1}-X\right)^{T} \\
& +X B_{p} B_{p}^{T} X+\delta_{1}^{-1} C_{p}^{T} C_{p} \\
& +\left(Y^{-1}-X\right) B_{c} B_{c}^{T}\left(Y^{-1}-X\right)^{T} .
\end{aligned}
$$

Furthermore, we have

$$
\left[\begin{array}{ll}
Q_{1} & Q_{2} \\
* & Q_{3}
\end{array}\right]=M_{1}+M_{2}+M_{3}+M_{4}<0
$$

(19) where

$$
\begin{aligned}
& M_{1}=\left[\begin{array}{cc}
A_{p} Y+B_{p} C_{c} Y & A_{p} \\
X A_{p} Y+X B_{p} C_{c} Y+\left(Y^{-1}-X\right) B_{c} C_{P} Y+\left(Y^{-1}-X\right) A_{c} Y & X A_{p}+\left(Y^{-1}-X\right) B_{c} C_{p}
\end{array}\right], \\
& M_{2}=\left[\begin{array}{cc}
Y A_{p}^{T}+Y^{T} C_{c}^{T} B_{p}^{T} Y A_{p}^{T} X+Y C_{c}^{T} B_{p}^{T} X+Y C_{P}^{T} B_{c}^{T}\left(Y^{-1}-X\right)^{T}+Y A_{c}^{T}\left(Y^{-1}-X\right)^{T} \\
A_{p}^{T}
\end{array}\right], \\
& M_{3}=\left[\begin{array}{cc}
B_{p} B_{p}^{T} & A_{p}^{T} X+C_{p}^{T} B_{c}^{T}\left(Y^{-1}-X\right)^{T} \\
* & X B_{p} B_{p}^{T} X+\left(\begin{array}{c}
\left.Y^{-1}-X\right) \\
B_{p} B_{p}^{T} X
\end{array}\right.
\end{array}\right], \\
& M_{4}=\left[\begin{array}{cc}
\delta_{1}^{-1} Y C_{p}^{T} C_{p} Y+\delta_{2}^{-1} Y^{T} C_{c}^{T} C_{c} Y & \delta_{1}^{-1} Y C_{p}^{T} C_{p} \\
* & \delta_{1}^{-1} C_{p}^{T} C_{p}
\end{array}\right] .
\end{aligned}
$$

Letting

$$
H=\left[\begin{array}{cc}
0 & I \\
Y^{-1} & -I
\end{array}\right]
$$

and premultiplying and postmultiplying LMI (22) by $H$ and $H^{T}$, we obtain

$$
H M_{1} H^{T}+H M_{2} H^{T}+H M_{3} H^{T}+H M_{4} H^{T}<0 .
$$


Since

$$
\begin{aligned}
& H M_{1} H^{T}=H\left[\begin{array}{cc}
A_{p} Y+B_{p} C_{c} Y & A_{p} \\
X A_{p} Y+X B_{p} C_{c} Y+\left(Y^{-1}-X\right) B_{c} C_{P} Y+\left(Y^{-1}-X\right) A_{c} Y & X A_{p}+\left(Y^{-1}-X\right) B_{c} C_{p}
\end{array}\right] H^{T} \\
& =H\left[\begin{array}{cc}
Y & Y \\
I & 0
\end{array}\right]\left[\begin{array}{cc}
X & Y^{-1}-X \\
* & X-Y^{-1}
\end{array}\right]\left[\begin{array}{cc}
A_{p} & B_{p} C_{c} \\
B_{c} C_{P} & A_{c}
\end{array}\right]\left[\begin{array}{cc}
Y & I \\
Y & 0
\end{array}\right] H^{T} \\
& =P \bar{A} \text {, } \\
& H M_{2} H^{T}=H\left[\begin{array}{cc}
Y A_{p}^{T}+Y^{T} C_{c}^{T} B_{p}^{T} & Y C_{P}^{T} B_{c}^{T}\left(Y^{-1}-X\right)^{T}+Y A_{c}^{T}\left(Y^{-1}-X\right)^{T}+Y A_{p}^{T} X+Y C_{c}^{T} B_{p}^{T} X \\
A_{p}^{T} & A_{p}^{T} X+C_{p}^{T} B_{c}^{T}\left(Y^{-1}-X\right)^{T}
\end{array}\right] H^{T} \\
& =H\left[\begin{array}{cc}
Y & Y \\
I & 0
\end{array}\right]\left[\begin{array}{cc}
A_{p}^{T} & C_{P}^{T} B_{c}^{T} \\
C_{c}^{T} B_{p}^{T} & A_{c}^{T}
\end{array}\right]\left[\begin{array}{cc}
X & Y^{-1}-X \\
* & X-Y^{-1}
\end{array}\right]\left[\begin{array}{cc}
Y & I \\
Y & 0
\end{array}\right] H^{T} \\
& =\bar{A}^{T} P \text {, } \\
& H M_{3} H^{T}=H\left[\begin{array}{cc}
B_{p} B_{p}^{T} & B_{p} B_{p}^{T} X \\
* & X B_{p} B_{p}^{T} X+\left(Y^{-1}-X\right) B_{c} B_{c}^{T}\left(Y^{-1}-X\right)^{T}
\end{array}\right] H^{T} \\
& =H\left[\begin{array}{cc}
I & 0 \\
X & Y^{-1}-X
\end{array}\right]\left[\begin{array}{cc}
0 & B_{p} \\
B_{c} & 0
\end{array}\right]\left[\begin{array}{cc}
0 & B_{c}^{T} \\
B_{p}^{T} & 0
\end{array}\right]\left[\begin{array}{cc}
I & X \\
0 & Y^{-1}-X
\end{array}\right] H^{T} \\
& =P \overline{B B}^{T} P \text {, } \\
& H M_{4} H^{T}=H\left[\begin{array}{cc}
\delta_{1}^{-1} Y C_{p}^{T} C_{p} Y+\delta_{2}^{-1} Y^{T} C_{c}^{T} C_{c} Y & \delta_{1}^{-1} Y C_{p}^{T} C_{p} \\
* & \delta_{1}^{-1} C_{p}^{T} C_{p}
\end{array}\right] H^{T} \\
& =H\left[\begin{array}{cc}
Y & Y \\
I & 0
\end{array}\right]\left[\begin{array}{cc}
\delta_{1}^{-1} C_{p}^{T} C_{p} & 0 \\
* & \delta_{2}^{-1} C_{c}^{T} C_{c}
\end{array}\right]\left[\begin{array}{cc}
Y & I \\
Y & 0
\end{array}\right] H^{T} \\
& =\left[\begin{array}{cc}
\delta_{1}^{-1} C_{p}^{T} C_{p} & 0 \\
* & \delta_{2}^{-1} C_{c}^{T} C_{c}
\end{array}\right] \text {, }
\end{aligned}
$$

we know that LMI (25) is equivalent to the following inequality

$$
P \bar{A}+\bar{A}^{T} P+P \bar{B} B^{T} P+\left[\begin{array}{cc}
\delta_{1}^{-1} C_{p}^{T} C_{p} & 0 \\
* & \delta_{2}^{-1} C_{c}^{T} C_{c}
\end{array}\right]<0
$$

which can be rewritten by

$$
\begin{aligned}
& x^{T}\left(P \bar{A}+\bar{A}^{T} P+P \bar{B} B^{T} P\right) x \\
& \quad+x^{T}\left[\begin{array}{cc}
\delta_{1}^{-1} C_{p}^{T} C_{p} & 0 \\
* & \delta_{2}^{-1} C_{c}^{T} C_{c}
\end{array}\right] x<0 .
\end{aligned}
$$

Since

$$
\begin{aligned}
e^{T} e & =e_{p}^{T} e_{p}+e_{c}^{T} e_{c} \leq \sigma_{1} y_{p}^{T} y_{p}+\sigma_{2} y_{c}^{T} y_{c} \\
& =x^{T}\left[\begin{array}{cc}
\sigma_{1} C_{p}^{T} C_{p} & 0 \\
0 & \sigma_{2} C_{c}^{T} C_{c}
\end{array}\right] x \\
& =x^{T}\left[\begin{array}{cc}
\delta_{1}^{-1} C_{p}^{T} C_{p} & 0 \\
* & \delta_{2}^{-1} C_{c}^{T} C_{c}
\end{array}\right] x,
\end{aligned}
$$

then we obtain that

$$
x^{T}\left(P \bar{A}+\bar{A}^{T} P+P \bar{B} B^{T} P\right) x+e^{T} e<0 .
$$

Combining (15) and (30), we have

$$
\dot{V}_{x}<0 \text {. }
$$

Hence, if LMIs (11) and (12) are satisfied, system (6) under the dynamic output feedback (2) with the matrices $A_{c}, B_{c}$, 
and $C_{c}$ and event generator (8) with $\sigma_{1}=\delta_{1}^{-1}, \sigma_{2}=\delta_{2}^{-1}$ is asymptotically stable, where

$$
\begin{aligned}
& A_{c}=-\left(Y-X^{-1}\right)\left(A_{P}+Y A_{p}^{T} X+V^{T} B_{p}^{T} X+Y C_{P}^{T} W^{T}\right. \\
& \left.+B_{p} B_{p}^{T} X+\delta_{1}^{-1} Y C_{p}^{T} C_{p}\right)^{T} Y^{-1}, \\
& B_{c}=\left(Y-X^{-1}\right) W \text {, } \\
& C_{c}=V Y^{-1} \text {. }
\end{aligned}
$$

The proof is completed.

Remark 3. Theorem 2 gives us a sufficient condition on how to co-design the event generator and the dynamic output feedback controller. Different from the co-design method in [16-18], this paper focuses on the dynamic output feedback. Moreover, the event-triggered condition in this paper also depends on the system output, which is much easier to realize with respect to the case depending on the system state.

Remark 4. For any given LTI model parameters $A_{p}, B_{p}, C_{p}$, we can calculate the inequalities (11) and (12) in Theorem 2. If the LMIs have feasible solutions $X, Y, V, W, \sigma_{1}, \sigma_{2}$, then we know system (6) is asymptotically stable. Furthermore, we obtain the parameters of the event generator and the controller. Therefore, the co-design problem is converted into the feasibility of LMIs, which is easy to be checked by using Matlab/LMI toolbox.

\section{A Numerical Example}

In this section, a numerical example is given to demonstrate the efficiency of the proposed method above. Consider the plant and its candidate dynamic output feedback controller as follows:

$$
\begin{gathered}
\dot{x}_{p}=\left[\begin{array}{cc}
0 & 1 \\
2 & -3
\end{array}\right] x_{p}+\left[\begin{array}{l}
2.2 \\
2.2
\end{array}\right] u_{p}, \\
y_{p}=\left[\begin{array}{ll}
-1 & 4
\end{array}\right] x_{p}, \\
\dot{x}_{c}(t)=A_{c} x_{c}(t)+B_{c} u_{c}(t), \\
y_{c}(t)=C_{c} x_{c}(t) .
\end{gathered}
$$

By solving the LMIs feasibility problem in Theorem 2, we have $X=\left[\begin{array}{cc}4.1064 & -4.4449 \\ -4.4449 & 5.4109\end{array}\right], Y=\left[\begin{array}{rr}19.3972 & 4.3338 \\ 4.3338 & 4.2859\end{array}\right], V=$ $\left[\begin{array}{ll}-9.1681-4.7274\end{array}\right] W=\left[\begin{array}{c}-1.8795 \\ 0.7450\end{array}\right], \delta_{1}=25.2983$, and $\delta_{2}=$ 28.0767 and then the parameters of the controller can be calculated as

$$
\begin{aligned}
& A_{c}=\left[\begin{array}{ll}
4.5499 & -18.8029 \\
5.7729 & -19.7737
\end{array}\right], \quad B_{c}=\left[\begin{array}{l}
4.2917 \\
3.5312
\end{array}\right], \\
& C_{c}=[-0.2922-0.8075] .
\end{aligned}
$$

From (8), we obtain that the event-triggered condition is

$$
e_{p}^{T} e_{p}+e_{c}^{T} e_{c} \leq 0.0395 y_{p}^{T} y_{p}+0.0356 y_{c}^{T} y_{c} .
$$

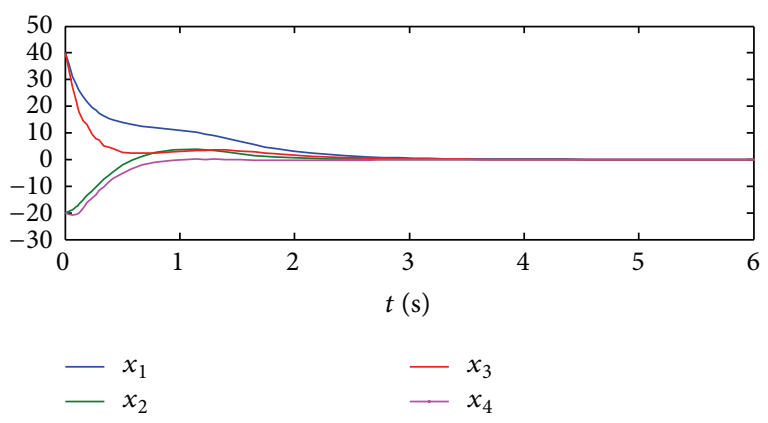

Figure 2: System state trajectories under the event-triggered dynamic output feedback controller.
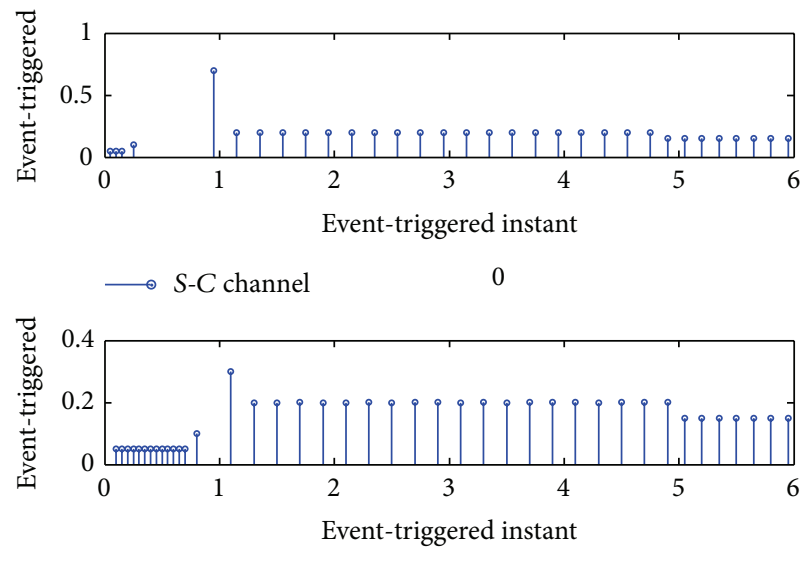

$\longrightarrow C$-A channel

FIGURE 3: Event-triggered instant and event-triggered interval.

Given the initial state $x_{0}=(40,-20,40,-20)^{T}$, we have the trajectory of the closed-loop system shown in Figure 2. It can be seen that the system is asymptotically stable.

Setting the sampling step as $0.05 \mathrm{~s}$, the effect of the event-triggered scheme from sensor to controller and from controller to actuator is shown in Figure 3, where the $x$ axis means the event-triggered instant and $y$-axis means the interval between the current event-triggered instant and the last event-triggered instant. After computation, the average sampling time from sensor to controller is $0.15 \mathrm{~s}$, which is 3 times that of the sampling time of the system if there is no event-triggered scheme; the average sampling time from controller to actuator is $0.18 \mathrm{~s}$, which is 3.6 times that of the sampling time of the system if there is no eventtriggered scheme. Therefore, the simulation shows that the system resource utilization is greatly saved by using the event-triggered scheme and the unnecessary waste of system resources is reduced.

\section{Conclusion}

In this paper, we studied the asymptotical stabilization of linear systems based on the event-triggered dynamic output feedback control. We proposed an approach to co-design the event generator and the dynamic output feedback controller. 
A sufficient condition was presented in terms of LMIs by using a quadratic Lyapunov function.

\section{Conflict of Interests}

The authors declare that there is no conflict of interests regarding the publication of this paper.

\section{Acknowledgments}

This work was supported by the National Natural Science Foundation of China under Grant 61233002 and the Fundamental Research Funds for the Central Universities under Grant N120404019.

\section{References}

[1] X.-M. Sun, G.-P. Liu, W. Wang, and D. Rees, "L2-gain of systems with input delays and controller temporary failure: zero-order hold model," IEEE Transactions on Control Systems Technology, vol. 19, no. 3, pp. 699-706, 2011.

[2] X. M. Sun, G. P. Liu, W. Wang, and D. Rees, "Stability analysis for networked control systems based on event-time-driven mode," International Journal of Control, vol. 82, no. 12, pp. 2260-2266, 2009.

[3] W. Zhang, M. S. Branicky, and S. M. Phillips, "Stability of networked control systems," IEEE Control Systems Magazine, vol. 21, no. 1, pp. 84-99, 2001.

[4] J. P. Hespanha, P. Naghshtabrizi, and Y. Xu, "A survey of recent results in networked control systems," Proceedings of the IEEE, vol. 95, no. 1, pp. 138-162, 2007.

[5] R. C. Dorf, M. Farren, and C. Phillips, "Adaptive sampling frequency for sampled-data control systems," IRE Transactions on Automatic Control, vol. 7, no. 1, pp. 38-47, 1962.

[6] K. J. Åström and B. Bo, "Comparison of periodic and event based sampling for first-order stochastic systems," in Proceedings of the 14th IFAC World Congress, Beijing, China, 1999.

[7] A. Karl-Erik, "A simple event-based PID controller," in Proceedings of the 14th IFAC World Congress, Beijing, China, 1999.

[8] E. Kofman and J. H. Braslavsky, "Level crossing sampling in feedback stabilization under data-rate constraints," in Proceedings of the 45th IEEE Conference on Decision and Control (CDC '06), pp. 4423-4428, December 2006.

[9] P. Tabuada, "Event-triggered real-time scheduling of stabilizing control tasks," IEEE Transactions on Automatic Control, vol. 52, no. 9, pp. 1680-1685, 2007.

[10] J. Mazo, A. Anta, and P. Tabuada, "An ISS self-triggered implementation of linear controllers," Automatica, vol. 46, no. 8, pp. 1310-1314, 2010.

[11] J. Lunze and D. Lehmann, "A state-feedback approach to eventbased control," Automatica, vol. 46, no. 1, pp. 211-215, 2010.

[12] M. C. F. Donkers and W. P. M. Heemels, "Output-based eventtriggered control with guaranteed $L_{\infty}$-gain and improved and decentralized event-triggering," IEEE Transactions on Automatic Control, vol. 57, no. 6, pp. 1362-1376, 2012.

[13] L. Li and M. Lemmon, "Event-triggered output feedback control of finite horizon discrete-time multi-dimensional linear processes," in Proceedings of the 49th IEEE Conference on Decision and Control (CDC '10), pp. 3221-3226, Atlanta, Ga, USA, December 2010.
[14] J. Almeida, C. Silvestre, and A. M. Pascoal, "Observer based selftriggered control of linear plants with unknown disturbances," in Proceedings of the American Control Conference (ACC '12), pp. 5688-5693, June 2012.

[15] P. Tallapragada and N. Chopra, "Event-triggered decentralized dynamic output feedback control for LTI systems," in Proceedings of the 3rd IFAC Workshop on Distributed Estimation and Control in Networked Systems (NECSYS '12), vol. 3, pp. 31-36, September 2012.

[16] P. Tallapragada and N. Chopra, "Event-triggered dynamic output feedback control for LTI systems," in Proceedings of the 51st IEEE Conference on Decision and Control (CDC '12), pp. 6597-6602, Maui, Hawaii, USA, December 2012.

[17] S. B. Li and B. G. Xu, "Co-design of event generator and controller for event-triggered control system," in Proceedings of the 30th Chinese Control Conference (CCC '11), pp. 175-179, July 2011.

[18] S. B. Li and B. G. Xu, "Event-triggered control for discrete-time uncertain linear parameter-varying systems," in Proceedings of the 32nd Chinese Control Conference, Xi'an, China, July 2013.

[19] S. Boyd, L. El Ghaoui, and E. F. Balakrishnan, Linear matrix inequalities in system and control theory, vol. 15, SIAM, Philadelphia, Pa, USA, 1994. 


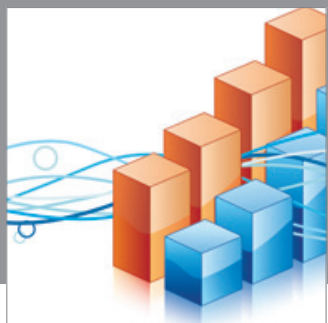

Advances in

Operations Research

mansans

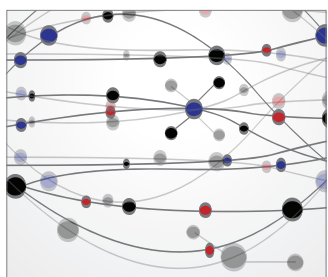

The Scientific World Journal
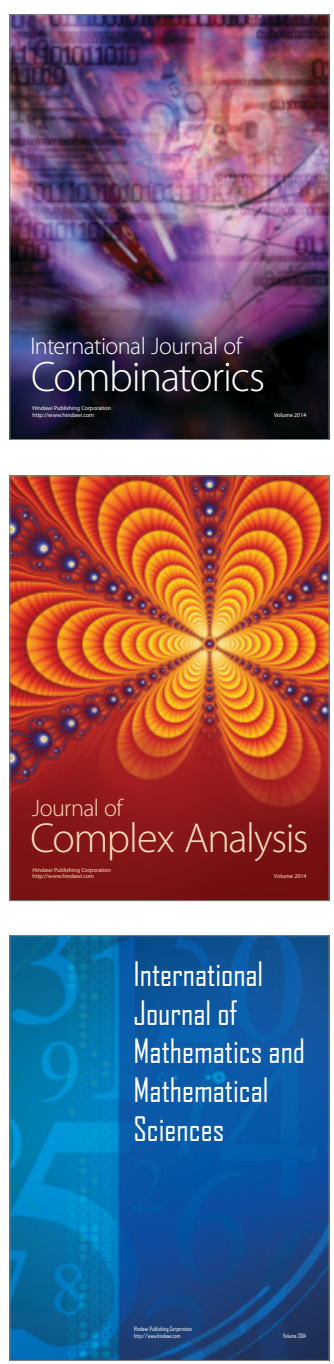
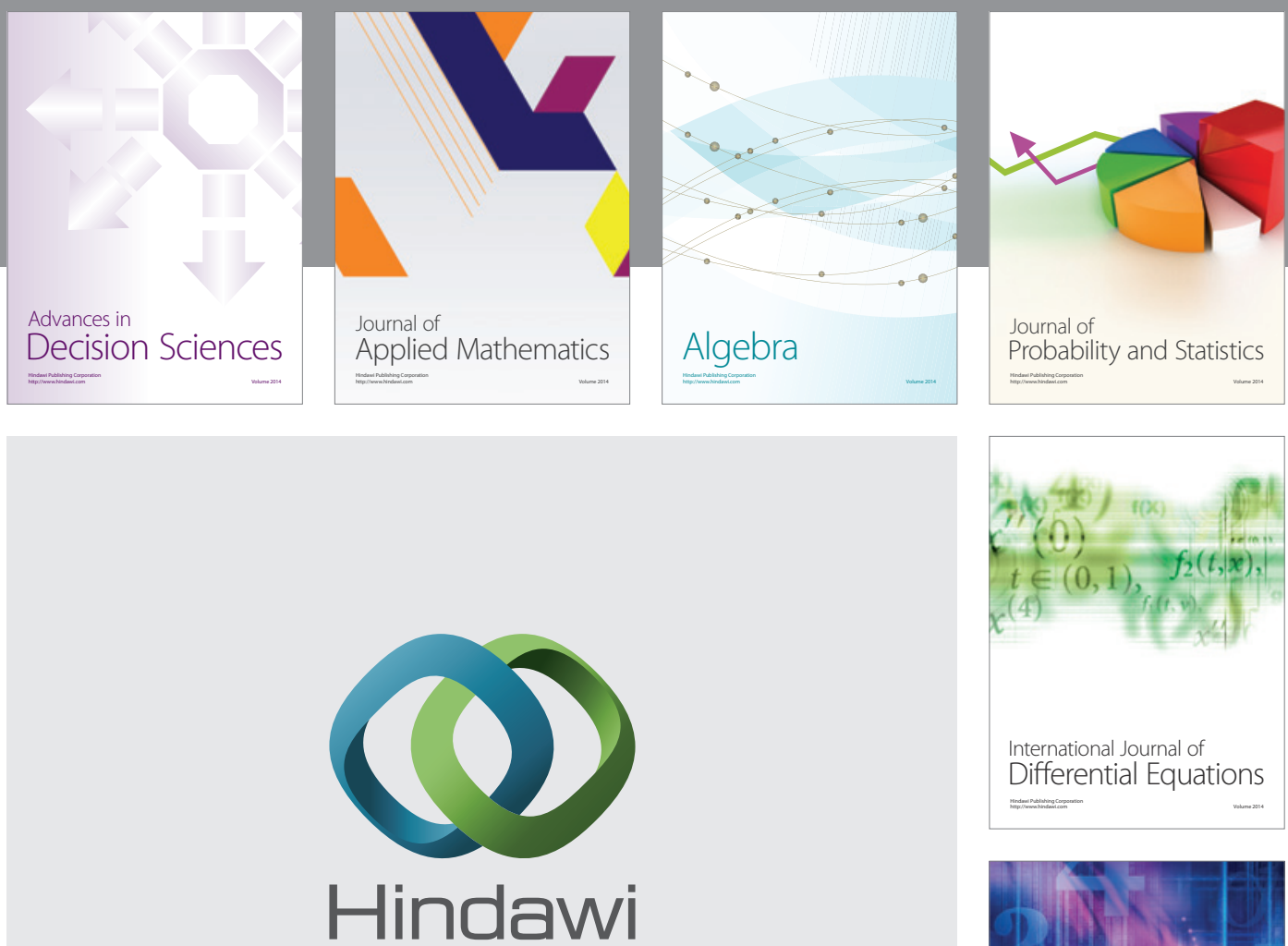

Submit your manuscripts at http://www.hindawi.com
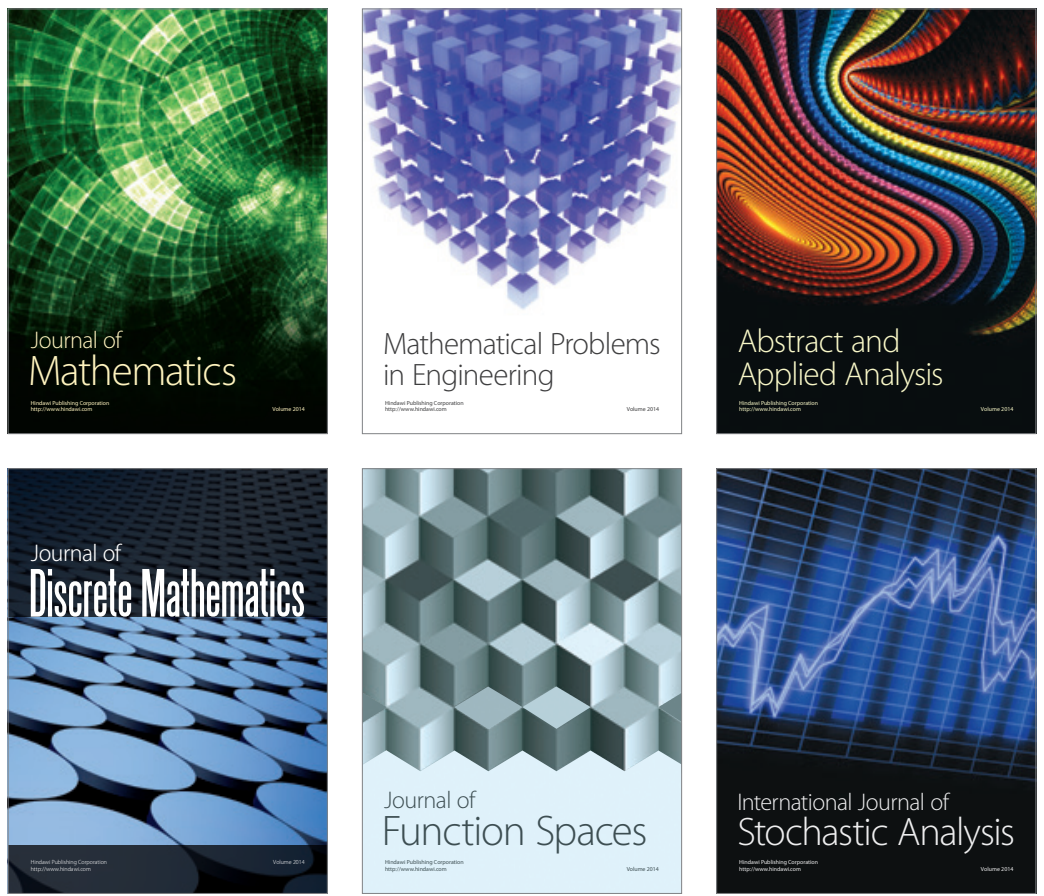

Journal of

Function Spaces

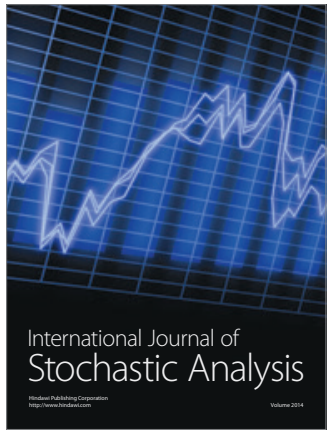

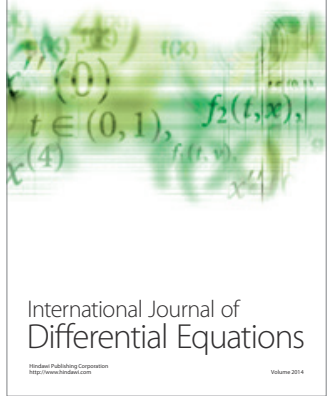
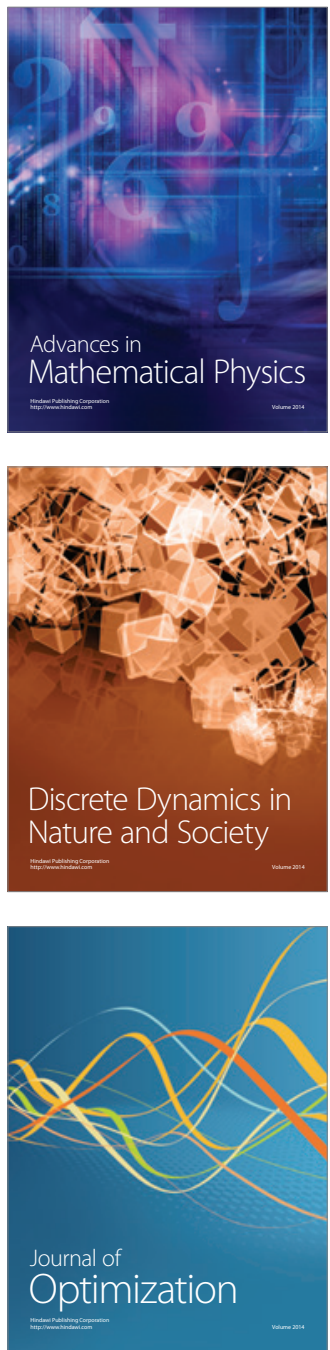